\title{
Evolution of Developmental Stages in Cultures of Bacillus cereus
}

\author{
DOROTHY K. FRASER AND JUDITH P. BAIRD \\ Department of Microbiology, Oregon State University, \\ Corvallis, Oregon, U.S.A.
}

(Accepted for publication 19 October 1967)

\begin{abstract}
SUMMARY
Cultures of Bacillus cereus grown in a chemically defined medium with glucose under standardized conditions followed a reproducible pattern of development giving good spore yields in less than $24 \mathrm{hr}$. Samples were withdrawn at intervals and individual organisms were classified into developmental states by four methods: (1) Gram reaction; (2) growth potential as revealed by the microcultures derived from single organisms or chains of organisms isolated into microdrops; (3) ability of individual organisms to take up neutral red; (4) electrophoretic distribution of organisms through a $\mathrm{pH}$ gradient. From the results we conclude that at most times the population was heterogeneous with respect to state of the organisms and that at least some of the shifts in state were quantal, in the sense that each state was relatively stable and that the transition from one state to the next rapid. There was a cyclical appearance and disappearance of organisms stainable with neutral red. Waves of stainable organisms were correlated with the break-up of chains into smaller units and with the lysis of a proportion of the organisms.
\end{abstract}

\section{INTRODUCTION}

In developing bacterial cultures there are progressive changes in the properties of the organisms and the culture medium produced by mutual interactions. We were interested to study the role of these interactions in determining morphogenesis of the organisms. The ideal approach to this problem would be to start from a complete description of the organism + medium system in every biochemical detail. At present a more realistic approach is to describe the states of individual organisms at various stages, to study the kinetics and mode of transition from state to state, and to identify the morphogenesis-determining interactions between the organisms and the medium without necessarily knowing their nature. We used this second approach in the model case of a culture of Bacillus cereus from spore germination through vegetative growth to sporulation. In work described in this paper, we describe changes in properties of the cultures and organisms in terms of a time scale.

\section{Organism and medium}

\section{METHODS}

The bacterial strain used was Bacillus cereus $569 \mathrm{R}$ from the collection of Dr C. B. Thorne. The growth medium (SMCA) contained $\left(\%\right.$, w/v): $0.025 \mathrm{~K}_{2} \mathrm{HPO}_{4}, 0.025$ $\mathrm{KH}_{2} \mathrm{PO}_{4}, 0.01 \mathrm{MgSO}_{4}, 0.0005 \mathrm{FeSO}_{4} \cdot 7 \mathrm{H}_{2} \mathrm{O}, 0.0005 \mathrm{MnSO}_{4} \cdot 4 \mathrm{H}_{2} \mathrm{O}, 0.5$ vitamin-free 
Casamino acids (Difco), 0.2 glucose, and ( $\mu \mathrm{g} . / \mathrm{ml}$.): 0.025 biotin, 0.25 niacin, $0.27 \mathrm{Ca}$ pantothenate. This medium was selected because it permits almost complete sporulation of B. cereus $569 \mathrm{R}$ within $24 \mathrm{hr}$. The germination medium consisted of $0.04 \mathrm{M}$-tris buffer $\left(\mathrm{pH} 8 \cdot 0\right.$ ) containing $0.00 \mathrm{I} \mathrm{M}-\mathrm{KH}_{2} \mathrm{PO}_{4}$ and $2.5 \mathrm{mg}$. L-alanine $/ \mathrm{ml}$.

\section{Culture conditions}

A washed heat-shocked suspension of spores in water ( $0 . \mathrm{I} \mathrm{ml}$. containing $2 \times 10^{8}$ spores as determined by microscopic counts and assays of viable heat-resistant spores) was heated at $65^{\circ}$ for $\mathrm{I} 5 \mathrm{~min}$. One $\mathrm{ml}$. of L-alanine germination medium was added, and the tube shaken at $37^{\circ}$ for $30 \mathrm{~min}$. The contents were added at $t=0 \mathrm{hr}$ to $50 \mathrm{ml}$. growth medium in a $250 \mathrm{ml}$. Erlenmeyer flask. Flasks were shaken at $37^{\circ}$ on a reciprocal shaker, and samples removed at desired intervals. When large samples were to be removed, duplicate cultures were used. Identifiable events occurred at reproducible times within $0.5 \mathrm{hr}$ for $t=0$ to $t=7 \mathrm{hr}$, and within $\mathrm{I} \mathrm{hr}$ for $t=7.5$ to $t=13 \mathrm{hr}$.

\section{Miscellaneous}

The extinction at $440 \mathrm{~m} \mu\left(E_{440}\right)$ was measured with a Zeiss PMQII spectrophotometer.

A Petroff-Hauser chamber was used for counts of organisms and chains. To count the individual organisms in chains, saturating sodium chloride was added to shrink the organisms (Powell, 1958). Counts were not accurate after $5 \mathrm{hr}$ because of clumping.

\section{Identification of states of organisms}

Organisms in different physiological or developmental states were scored on the basis of four properties.

(I) Type of development of individual organisms. A small drop of mineral oil was placed on a microscope slide. A sample of the culture was diluted when necessary into fresh SMCA medium to about $5 \times 10^{6}$ organisms or chains $/ \mathrm{ml}$. With a pipette drawn from capillary glass tubing a pattern of 100 droplets (volume about $10^{-7} \mathrm{ml}$. each) was placed on the glass under the oil by the use of a dissecting microscope; a coverslip was then placed on the oil drop. Most droplets contained either one organism or a chain or none. The slides were studied microscopically to locate organisms or chains, incubated for 2 days at $37^{\circ}$ in a moist chamber, and the droplets scored for the following types of development: $(a)$ no growth, $(b)$ division without spore formation, $(c)$ division with spore formation, $(d)$ spore formation without division. The results served to calculate the frequency of organisms which gave each type of development, by using the Poisson distribution.

(2) Staining with neutral red. An aqueous solution of neutral red $(0.85 \%$, w/v) was used to stain living organisms in samples of the developing culture with I vol. dye solution to 4 vol culture. The organisms were examined by phase-contrast microscopy and photographed under oil immersion with $35 \mathrm{~mm}$. Kodak Plus-X or Panatomic-X film. Individual organisms were scored as bright (stained), dark (unstained), or bright with dark nuclear structures.

(3) Gram reaction. A heated-fixed smear was stained with $0.5 \%$ (w/v) crystal violet for I min., drained, treated with Gram iodine solution for I min., washed with $95 \%(\mathrm{v} / \mathrm{v})$ ethanol in water, rinsed with water and dried. 
(4) Distribution through a $\mathrm{pH}$ gradient. The method of electrophoretic separation of bacteria on the basis of their isoelectric points was adapted from Kolin (1958). The apparatus was constructed of glass tubing of the same dimensions as in the Kolin apparatus.

The gradient $\mathrm{pH} 7.5$ to 3.5 was established with $0.1 \mathrm{M}$-tris $\mathrm{HCl}$ buffers, with $2 \mathrm{M}$-sorbitol to make the dense acidic buffer. Sorbitol was used to stabilize the gradient. The procedure for filling the $U$ tube and forming the ' $M$ layer' (the region containing the sample) was as described by Kolin (1958). A millimetre scale was attached to one arm of the $U$ tube and the ' $M$ layer' was formed in $I \mathrm{~cm}$. in the centre of this scale. The samples were either concentrated by centrifugation followed by resuspension with supernatant fluid or diluted with SMCA medium to give an extinction $E_{440}$ of about $0 \cdot 6$ after an equal volume of 2 M-sorbitol had been added. All solutions were chilled in ice before use to decrease the effect of heat on the organisms during electrophoresis. The glass tubes containing the platinum electrodes were connected to the $U$ tube chamber by filter-paper wicks. With a Beckman Duostat power supply, current was applied for I min. at $2 \mathrm{~mA}$. and increased to $10 \mathrm{~mA}$., I50 V. for a total of $20 \mathrm{~min}$. Bands of organisms formed in the 'M layer' did not change position after this time. Organisms were scored by band position in the $\mathrm{pH}$ gradient.

\section{RESULTS}

Once a set of parameters (initial medium, volume, temperature, aeration, inoculum) is chosen, the course of development of a culture is completely determined. The changes in state of the organisms are not directly dependent on time, but are determined by a number of other variables which are altered by the growth of the organisms (population density, $\mathrm{pH}$ value of the medium, nutrient supply and other changes of the medium). With a single set of parameters, all these dependent variables follow a reproducible pattern, and the time of incubation may be used to define the state of the culture at any instant.

\section{Description of the culture properties}

Figure I gives data obtained by sampling from a standard culture (see Methods) for the period from $t=2$ to $t=13 \mathrm{hr}$ of incubation. The extinction of samples removed at intervals from the growing culture indicated progressively decreasing growth rates. During the stationary phase a reproducible decrease in $E_{440}$ occurred at $t=9.5 \mathrm{hr}$. The increases in counts of organisms corresponded closely to increases of extinction. The apparent breaks in the continuous curve of the count coincided with periods of lysis (see below), but may have been due to inaccuracies of counting. Bacillus cereus characteristically grows in chains. The first one or two divisions resulted in organisms which then separated; chain formation began after incubation for about $3 \mathrm{hr}$. The chains broke up into smaller units mainly at two times, $t=5 \mathrm{hr}$ and $t=7 \mathrm{hr}$. After $7 \mathrm{hr}$, growth was mainly in chains of 2 to 4 organisms. The $\mathrm{pH}$ value of the standard culture medium dropped to a low point at about $t=5 \mathrm{hr}$, gradually rose until about $t=\mathrm{Io} \mathrm{hr}$, and then continued near a maximum of $\mathrm{pH} 7 \cdot 4$. The decrease in $\mathrm{pH}$ value followed by a rise has been observed by other workers with different sporulation media (Halvorson, 1957). 


\section{Classification of states of organisms}

To study the developmental changes in the organisms during growth it was necessary to use tests by which individual organisms (rather than the culture as a whole) could be classified according to their developmental or physiological states. The use of such tests permitted us to determine whether organisms changed state in a gradual or in a sudden, quantal manner, and whether the population consisted of organisms which were all in one state of development at any given time, or whether it was heterogeneous. Four such techniques were used.

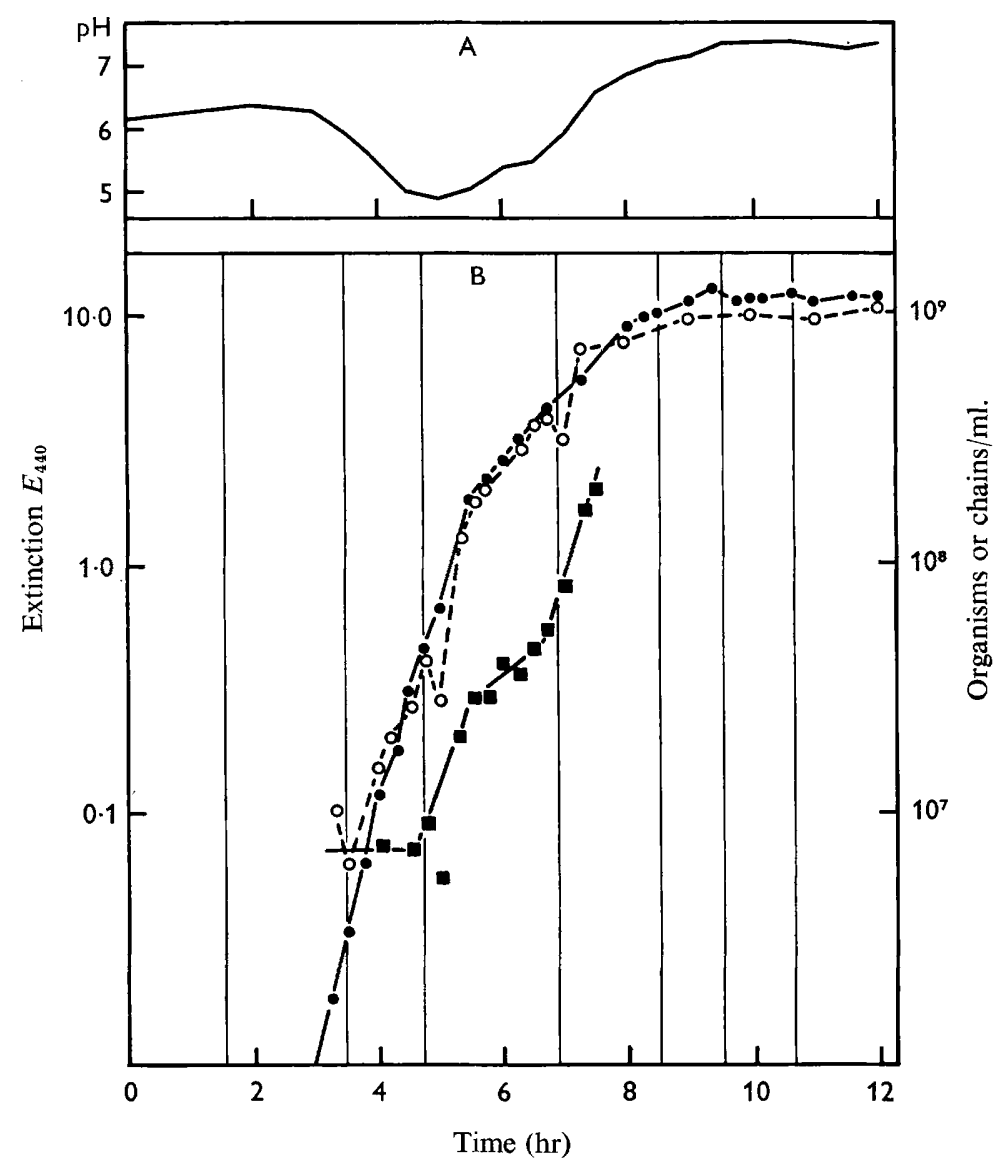

Fig. I. Bacillus cereus 569 R. A. Changing pH during culture development. B. Growth of standard culture. $(\bullet)$ extinction, $(O)$ organism counts, $(\square)$ chain counts.

\section{Gram reaction}

Samples of the standard culture were removed at 5 min. intervals and stained by the Gram method. All the organisms in samples taken before $t=3.5 \mathrm{hr}$ were Gramnegative. During the period from $t=3.5$ to $3.75 \mathrm{hr}$, all the organisms uniformly became more darkly stained; after $t=3.75 \mathrm{hr}$. all the organisms were Gram-positive (Fig. 2, top). 
Staining with neutral red

Samples taken at intervals from the culture were stained with aqueous neutral red, examined microscopically (phase contrast), and representative fields photographed. Stained organisms appeared refractile (bright) while unstained organisms or parts of organisms were dark. In some organisms dark bodies or complex structures, probably nuclear material, were seen in bright cytoplasm.

(a) Germination and outgrowth. Even after pre-treatment with heat and L-alanine the spores did not germinate simultaneously in the standard culture. The events occurring during the first $2 \mathrm{hr}$ are complex and will be dealt with in a separate paper. Plate $\mathrm{I}$, fig. 3 and 4 , show stages in this process. At $\mathrm{I} \cdot 5 \mathrm{hr}$ (Pl. I, fig. $3 b$ ) the outgrowing organisms were stainable with neutral red and appear bright under phase contrast. At $2-2 \cdot 5 \mathrm{hr}$ some organisms were seen to disintegrate or lose their contents (Pl. I, fig. 4).

(b) Periodicity in the frequency of bright organisms. During the period from $t=\mathrm{I}$ to $t=\mathrm{I} 2 \mathrm{hr}$, neutral red staining of samples taken at intervals showed that bright organisms appeared in 7 successive waves, reaching their peaks at the following times: $t=\mathrm{I} \cdot 5,3 \cdot 5,4 \cdot 75,6 \cdot 75,8 \cdot 5,9 \cdot 5,10 \cdot 75 \mathrm{hr}$, respectively. These peaks are indicated by vertical lines and numbered at the top of Fig. 2. Except for the first peak, at $\mathrm{I} \cdot 5 \mathrm{hr}$, the maximum proportion of bright organisms did not exceed $30 \%$ of the total number of organisms, the rest remaining dark. Counts of stained organisms were made in detail for peak no. 4 and are plotted on Fig. 2.

The bright organisms appeared either within dark chains (Pl. I, fig. 7; Pl. 2, fig. Io), or in chains consisting entirely of bright organisms, or in chains composed of bright organisms and organisms with bright cytoplasm and dark nuclei (Pl. 2, fig. Io). In the rising phase of each wave, fully bright organisms in the dark chains appeared in groups of one, two, or three with about equal frequency. This is a non-clonal and non-random distribution. Very few intermediately bright organisms were observed. In the falling phase of each wave, the brightness of the organisms slowly faded (Pl. I, fig. 5; Pl. 2, fig. II $b$ ) and internal structure could sometimes be observed (Pl. 2, fig. 9). Between waves the organisms appeared uniformly dark (Pl. I, fig. 6).

(c) Lytic phenomena. Soon after the number of bright organisms in any given wave reached a maximum, microscopic observation showed that some organisms were disintegrating, or cell material was being extruded through the cell wall at one or more places (Pl. I, figs. 4, 5; Pl. 2, figs. 8, I I $a$ ). The times at which this apparent lysis was observed are marked by arrows on Fig. 2. Lysis was observed also in unstained organisms (Pl. 2, fig. 8).

\section{Growth potential.}

Single organisms or chains isolated in microdrops under oil were allowed to develop at $37^{\circ}$ for 2 days (see Methods). Among organisms taken from the standard culture at times from 2 to $\mathrm{I} 3 \mathrm{hr}$ after inoculation, four clearly distinct types of development were observed. Five cell 'states' $(\alpha-\epsilon)$ were inferred from these results as indicated in Table $\mathrm{I}$.

Organisms in state $\alpha$ were usually single and divided only once if at all; chains in state $\beta$ grew into clones of about I0o organisms and no spores were formed by the progeny. Chains in state $\gamma(2-14$ organisms) also formed clones of about 100 organisms and usually all of these progeny formed spores. Occasionally, a single very long chain of unsporulated organisms was present among hundreds of free spores in the 
same microdrop. Organisms in state $\delta$ were a minority group of very short chains (about 2 organisms) which did not develop. Organisms or short chains in state $\epsilon$ ( $\mathrm{I}-4$ organisms) did not divide but formed spores in the original organisms. Samples of 100 microdrops containing organisms taken from the developing culture at any given time showed that the organisms were not usually all in the same state. This heterogeneity is shown by the overlapping curves $\alpha-\epsilon$ in Fig. 2.

It is likely that oxygen was limiting in the microdrops, and this may account in part for the fact that organisms in different states show different types of development. Halvorson (1957) showed that a sharp peak in oxygen demand followed the low $\mathrm{pH}$ point in sporulation cultures of Bacillus cereus. In our system this peak would correspond to the time at which the organisms shifted from state $\beta$ to state $\gamma$. Therefore, if ample oxygen were not available in the microdrops, the organisms may not have been able to make the shift.

\section{Table I. Bacillus cereus 569 R. Definition of developmental states of organisms in culture}

Single organisms or chains were isolated from a standard culture into microdrops. Types of growth were observed after incubation for $48 \mathrm{hr}$.

$\begin{array}{cccc}\begin{array}{c}\text { State } \\ \text { of } \\ \text { organism }\end{array} & \begin{array}{c}\text { Time of appearance } \\ \text { in standard culture } \\ (\mathrm{hr})\end{array} & \overbrace{\text { Clone formation }}^{\text {Growth observed in microcultures }} \\ \alpha & 2-4 & - & - \\ \beta & 3-6 & + & - \\ \gamma & 4 \cdot 5-13 & + & + \\ \delta & 7 \cdot 5-13 & - & - \\ \epsilon & 9 \cdot 5-13 & - & +\end{array}$

The interpretation of these findings would be clearer if individual organisms were used instead of chains. We were unable to break the chains successfully by ultrasonic treatment. Nevertheless, the sharp distinctions between the types of growth resulting from individual chains isolated into microdrops means that the states were sharply differentiated and did not grade into each other.

\section{Correlation of observed events with a time-scale}

Figure 2 shows the data, described above, in terms of a time-scale. In this figure, as well as in Fig. I, vertical lines have been drawn through the times at which peaks of bright organisms were observed, as numbered at the top of Fig. 2. Some lysis was noted microscopically after each peak of bright organisms, at the times indicated by arrows in Fig. 2. The curve of visible organisms counts (Fig. I) shows decreases in numbers following bright organism peaks 2, 3 and 4 , and a decrease in extinction occurred after peak 6. A break-up of the chains into smaller units followed peaks 3 and 4 (Fig. I). These correlated events seem to show in the development of the culture a periodicity not directly related to the division cycle. The shift from Gram-negativity to Gram-positivity occurred at the time of peak 2 (Fig. 2) and the low $\mathrm{pH}$ point of the medium at the time of peak 3 (Fig. I). The numbers of organisms in each development state, as determined by their growth potential in microdroplets, is plotted against time in Fig. 2. The overlapping curves $\alpha-\epsilon$ illustrate the heterogeneity observed in the culture at each time. The shifts in state of organisms do not appear to occur at times correlated in any simple way with the periodic phenomena listed above, except possibly for the small group of non-viable $\delta$ organisms. 


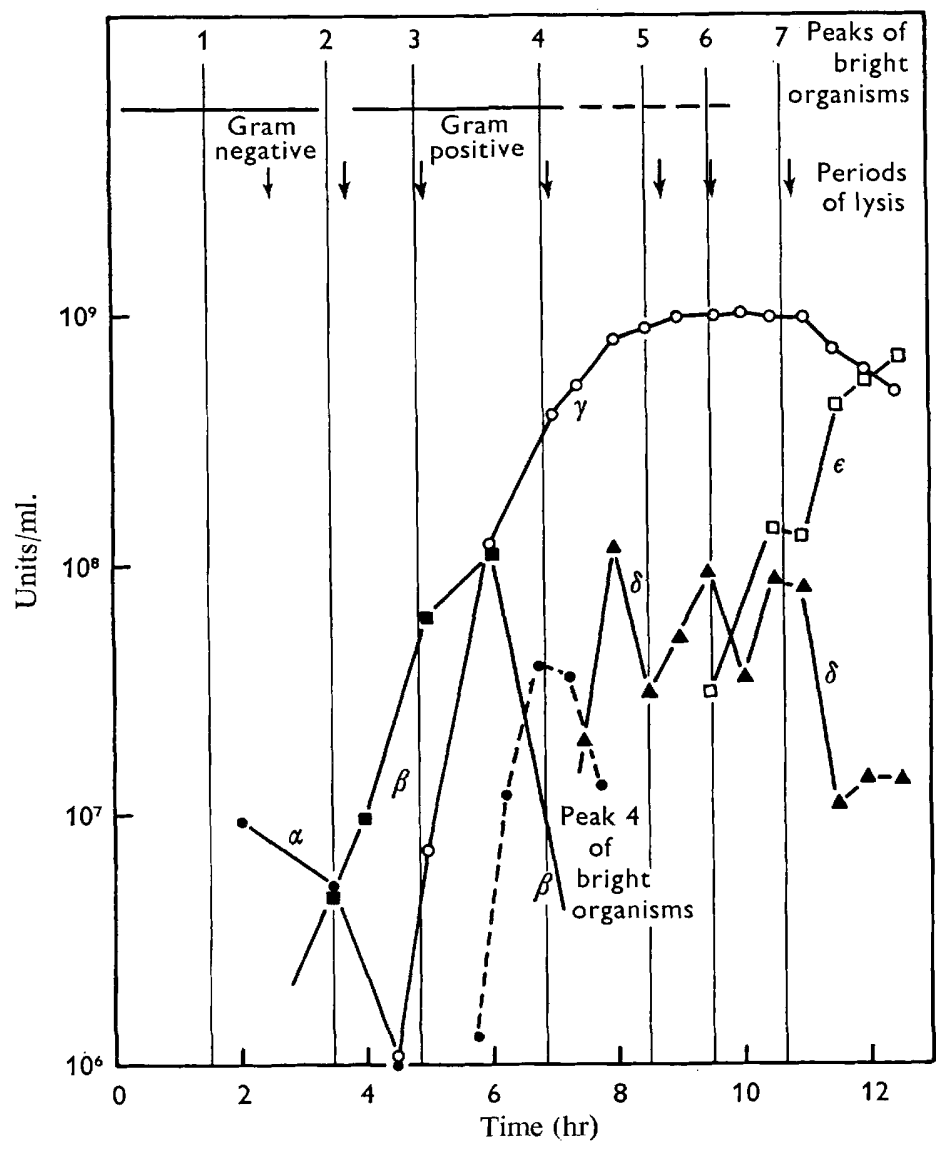

Fig. 2. Bacillus cereus 569 R. Population changes in organism state during development. Upper line, numbers indicate peaks of organisms which appeared bright when stained with neutral red; second line, Gram reaction; third line, arrows indicate periods of lysis. Solid curves (๑) organisms in state $\alpha ;(\boldsymbol{\square})$ in state $\beta ;(0)$ in state $\gamma ;(\boldsymbol{\Delta})$ in state $\delta ;(\square)$ in state $\epsilon$. Dashed curve, organisms staining with neutral red in peak 4 .

\section{Classification of types of organisms by isoelectric point}

Samples of the culture taken at different times were subjected to electrophoresis through a $\mathrm{pH}$ gradient stabilized with sorbitol (see Methods). This technique determines the range of isoelectric points of the organisms in a suspension and is not a measure of their electrophoretic mobility (Kolin, 1958). The purpose of the experiment was to determine whether the population at any one time was heterogeneous in isoelectric point, whether there were discrete groups of points or whether the isoelectric points varied over a continuous range. The results are shown in Fig. 3. It is clear that the organisms were grouped into 4 to 6 distinct bands. These bands are either sharp (groups of organisms with similar isoelectric points) or wide (groups of organisms or chains with isoelectric points continuously varying within a range). Repeated experiments gave results in general agreement but somewhat variable in detail. At certain times, bluish bands containing cellular debris but no intact organisms 
were observed at the top of the gradient. These probably represented the products of the disintegration of organisms as seen microscopically.

Organisms were recovered with a micropipette from the major bands and tested for staining with neutral red. Each band consisted of organisms which stained alike, but the staining property was not correlated with the position of the band in the gradient and did not correspond to the staining ability of the organisms in the growing culture, since no mixed chains were observed.

The results permit the clear conclusion that at any one time there existed within the culture 4 to 6 groups of organisms which differed discontinuously in isoelectric point. The groups with a range of isoelectric points may have been due to mixed chains, such as those of the bright and dark organisms observed by neutral red staining. The isoelectric range of each sample indicated a greater complexity of the system than was obvious from the distribution experiments or the neutral red staining.
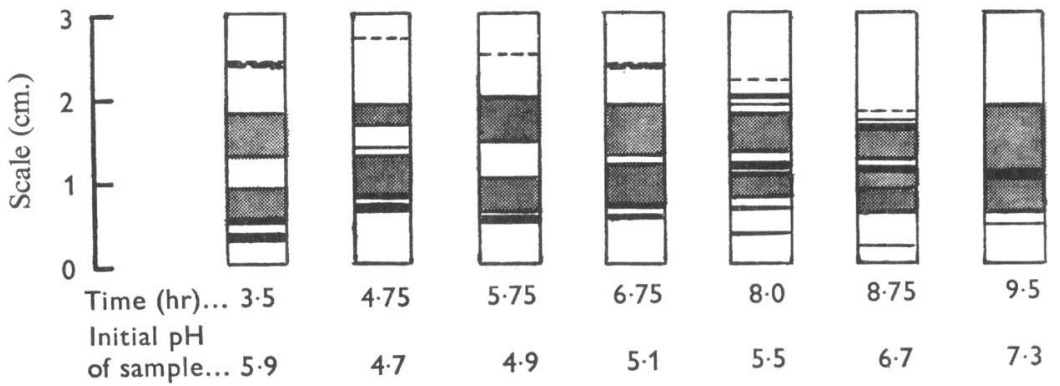

Fig. 3. Isoelectric ranges of samples from developing culture of Bacillus cereus $569 \mathrm{R}$. Diagram of location of bands after $20 \mathrm{~min}$. electrophoresis at $10 \mathrm{~mA}$, $150 \mathrm{~V}$. Sample initially occupied area between I and $2 \mathrm{~cm}$. $\mathrm{pH}$ of sample was determined after addition of sorbitol. Initial $\mathrm{pH}$ at $\mathrm{o} \mathrm{cm} ., 3 \cdot 3-3 \cdot 5$; initial $\mathrm{pH}$ at $3 \mathrm{~cm} ., 7 \cdot 5$. Bands indicated by dashes did not contain whole organisms.

\section{DISCUSSION}

A developing culture goes through a series of phases each of which is conditioned by the previous phases and in turn conditions the following ones. The process probably involves both intracellular changes, including cell division, and extracellular (medium) changes produced by the previous growth history of the culture. In this paper evidence is given that in these cultures individual organisms underwent quantal changes in shifting from one state to the next. In the case of the Gram reaction, all organisms shifted simultaneously and relatively suddenly from Gram-negative to Gram-positive. Since the organisms were not growing synchronously, this shift must have occurred in response to an environmental change. The rapidity of the shift suggests that this was a threshold reaction of the organisms to a more gradually changing environmental variable.

The growth potential tests show two distinct phenomena. First, a number of individuals taken from the culture at any given time and isolated into similar microdrops differ from each other with respect to their subsequent growth. This means that the sampled population was heterogeneous, and that the future behaviour of each organism when placed in the uniform environment of the microdrop had already been determined by its previous interactions in a spatially uniform but temporally 
changing environment. Secondly, each state of the organisms was sharply defined by the type of growth resulting from single organisms or chains. Organisms in transition from state to state would be expected to produce intermediate types of growth. These were not observed and must be rare. Therefore, the shifts in state must occur rapidly in individual organisms. The isoelectric range experiments also showed that distinct groups of organisms existed within the culture at any given time. The separation of bands confirms that organisms in intermediate stages were rare and indicates that the shifts in isoelectric point must be rapid.

With neutral red staining during the appearance of bright organisms, transition stages from dark to bright were rare, again indicating that changes in state occurred rapidly. After the peak frequencies of bright organisms had been reached, however, the changes from bright to dark occurred gradually.

The bright organisms appeared in cyclical waves during the growth of the culture. This oscillatory behaviour presumably means that the first individuals which gave a certain response caused a new change of environment which altered the reaction of other individuals. Since after each wave a fraction of the organisms lysed, the substances so released may play a role in controlling the oscillation in frequency of bright organisms.

With many transformable strains of bacteria, competent organisms appear towards the end of logarithmic growth (Schaeffer, 1964), at the time at which wave 4 of bright organisms was observed in our cultures of Bacillus cereus. Occasionally, successive waves of competency have been observed (Thomas, 1955). Jensen \& Haas (1963) reported correlation between the capacity for uptake of methylene blue and the presence of competent bacteria in a culture. Even through B. cereus $569 \mathrm{R}$ has not been shown to be transformable it seems possible that in transformable strains competency may be coupled with changes in stainability like those revealed by the present experiments with neutral red.

The authors thank Dr S. E. Luria for assistance in the preparation of the manuscript and Drs Harlyn O. Halvorson and Marguerite Vogt for useful suggestions. We gratefully acknowledge financial support from training grant 5T I AI I86-05 from the National Institute of Allergy and Infectious Diseases and from grant GB-4556 from the National Science Foundation (U.S.A.).

\section{REFERENCES}

Halvorson, H. O. (1957). Rapid and simultaneous sporulation. J. appl. Bact. 20, 305.

JENSEN, R. A. \& HAAS, F. (1963). Electrokinetics and cell physiology. J. Bact. 86, 73.

Kolin, A. (1958). Rapid electrophoresis in density gradients combined with pH and/or conductivity gradients. Meth. biochem. Analysis 6, 259.

Powell, E. O. (1958). An outline of the pattern of bacterial generation times. J. gen. Microbiol. I8, 382 .

SChaEfFER, P. (1964). Transformation. In The Bacteria. Ed. by I. C. Gunsalus and R. Y. Stanier, vol. 5, p. I 16. New York and London: Academic Press.

Thomas, R. (1955). Recherches sur la cinétique des transformations bactériennes. Biochem. biophys. Acta $\mathbf{1 8}, 467$. 


\section{EXPLANATION OF PLATES}

Figs. 3-11. Successive changes in morphology of organisms in the developing culture of Bacillus cereus $569 \mathrm{R}$. The organisms were stained with aqueous neutral red except for Pl. 2, fig. 6. The organisms were photographed under phase contrast at a magnification of $\times$ I 450 . Stained organisms appeared bright; unstained organisms appeared dark.

\section{Plate I}

Fig. 3. Outgrowth. The organisms were concentrated by centrifugation. (a) I.० hr; (b) I.5 hr; (c) $2 \cdot 7 \mathrm{hr}$.

Fig. 4. Early growth and lysis, (a) Swollen pair, $2 \cdot 75 \mathrm{hr},(b)$ lysis of bright organisms, $2 \cdot 5 \mathrm{hr}$; (c) growth and lysis of dark organisms, $2.5 \mathrm{hr}$.

Fig. 5. Lysis, (a) leakage from fading bright chain, $4.0 \mathrm{hr} ;(b)$ lysis, $4.0 \mathrm{hr} ;(c)$ lysis, $4.8 \mathrm{hr}$.

Fig. 6. Dark organisms, $4 \cdot 5 \mathrm{hr}$.

Fig. 7. Mixed chains, one intermediate organism, $4 \cdot 8 \mathrm{hr}$.

Fig. 8. Lysis, $5^{\circ} \circ \mathrm{hr}$. Unstained.

\section{Plate 2}

Fig. 9. Complex structures in fading organisms, $5 \cdot 75 \mathrm{hr}$.

Fig. IO. Bright organism distribution. (a) and (b) $6 \mathrm{hr}$; (c) $6.25 \mathrm{hr}$.

Fig. I I. (a) Lysis of bright organism, $7 \mathrm{hr}$; (b) fading of bright organisms, $7 \mathrm{hr}$. 

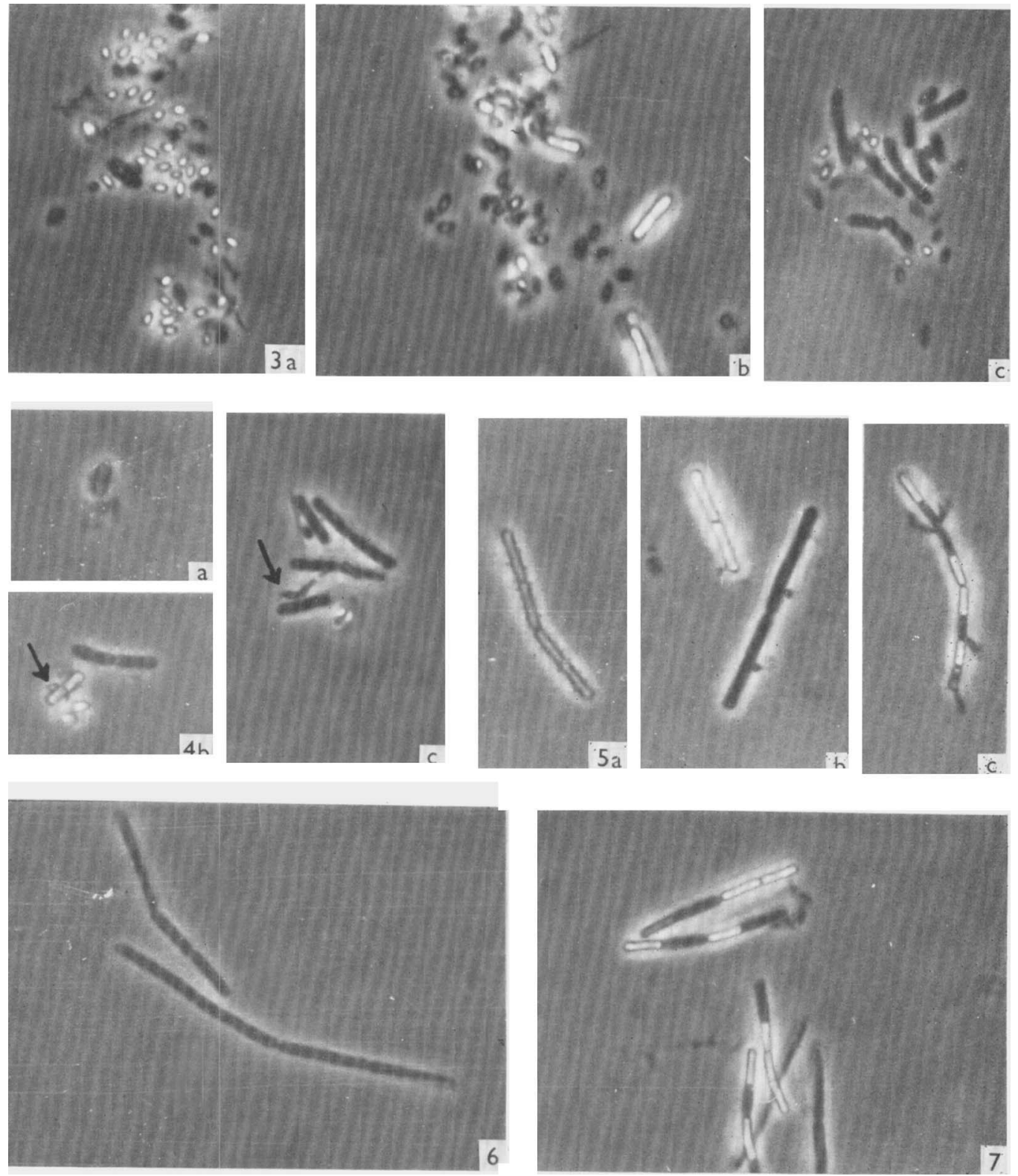

D. K. FRASER AND J. P. BAIRD

(Facing p. 402) 
Journal of General Microbiology, Vol. 5I, No. 3

Plate 2
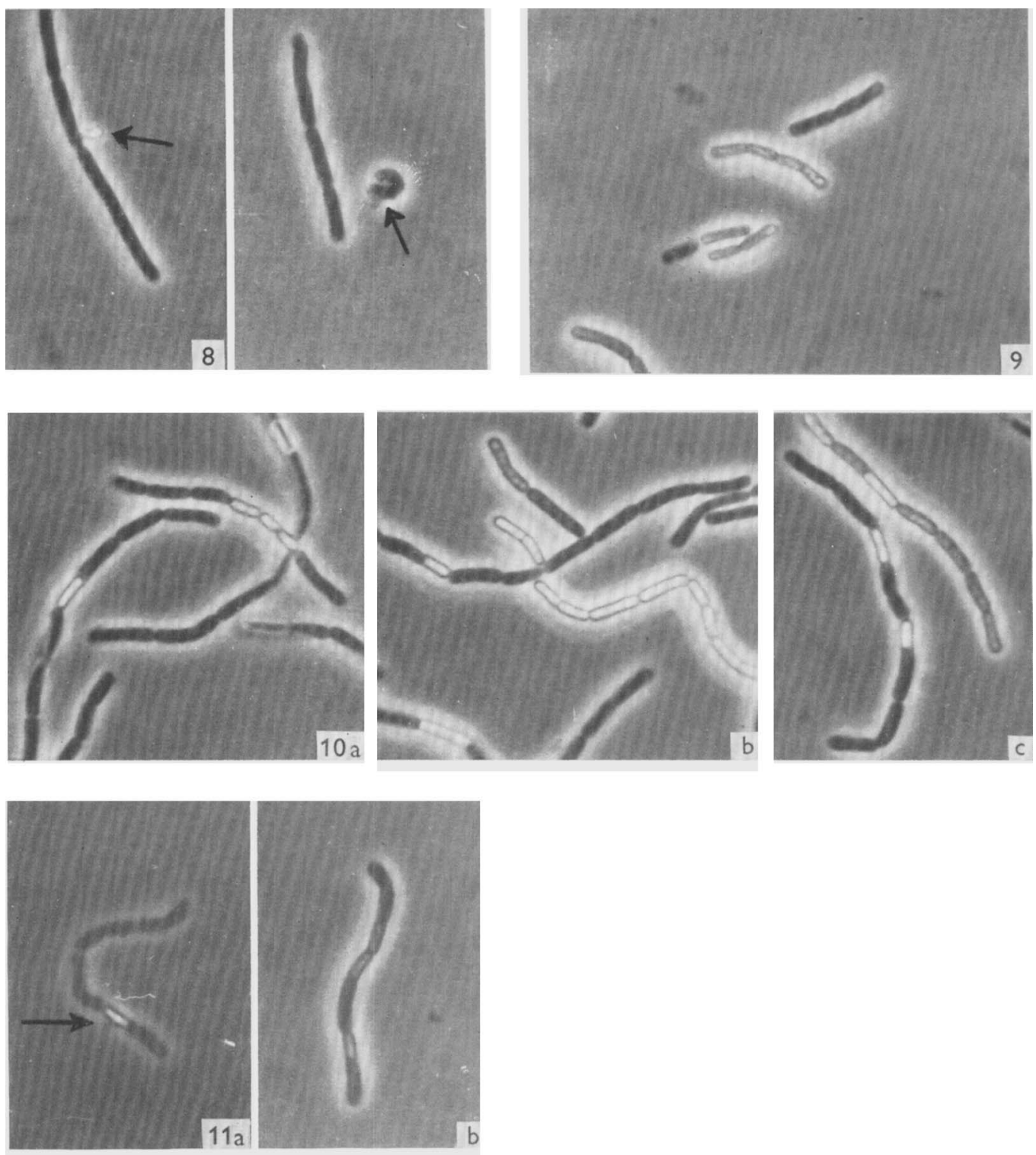

D. K. FRASER AND J. P. BAIRD 